\title{
Ground state homoclinic orbits of damped vibration problems
}

\section{Guan-Wei Chen ${ }^{*}$ and Jian Wang}

"Correspondence: guanweic@163.com

School of Mathematics and Statistics, Anyang Normal University, Anyang, Henan 455000, P.R. China

\begin{abstract}
In this paper, we consider a class of non-periodic damped vibration problems with superquadratic nonlinearities. We study the existence of nontrivial ground state homoclinic orbits for this class of damped vibration problems under some conditions weaker than those previously assumed. To the best of our knowledge, there has been no work focused on this case.
\end{abstract}

MSC: 49J40; 70H05

Keywords: non-periodic damped vibration problems; ground state homoclinic orbits; superquadratic nonlinearity

\section{Introduction and main results}

We shall study the existence of ground state homoclinic orbits for the following nonperiodic damped vibration system:

$$
\ddot{u}(t)+M \dot{u}(t)-L(t) u(t)+H_{u}(t, u(t))=0, \quad t \in \mathbb{R},
$$

where $M$ is an antisymmetric $N \times N$ constant matrix, $L(t) \in C\left(\mathbb{R}, \mathbb{R}^{N \times N}\right)$ is a symmetric matrix, $H(t, u) \in C^{1}\left(\mathbb{R} \times \mathbb{R}^{N}, \mathbb{R}\right)$ and $H_{u}(t, u)$ denotes its gradient with respect to the $u$ variable. We say that a solution $u(t)$ of (1.1) is homoclinic (to 0$)$ if $u(t) \in C^{2}\left(\mathbb{R}, \mathbb{R}^{N}\right)$ such that $u(t) \rightarrow 0$ and $\dot{u}(t) \rightarrow 0$ as $|t| \rightarrow \infty$. If $u(t) \not \equiv 0$, then $u(t)$ is called a nontrivial homoclinic solution.

If $M=0$ (zero matrix), then (1.1) reduces to the following second-order Hamiltonian system:

$$
\ddot{u}(t)-L(t) u(t)+H_{u}(t, u(t))=0, \quad t \in \mathbb{R} .
$$

This is a classical equation which can describe many mechanic systems such as a pendulum. In the past decades, the existence and multiplicity of periodic solutions and homoclinic orbits for (1.2) have been studied by many authors via variational methods; see [1-18] and the references therein.

The periodic assumptions are very important in the study of homoclinic orbits for (1.2) since periodicity is used to control the lack of compactness due to the fact that (1.2) is set on all $\mathbb{R}$. However, non-periodic problems are quite different from the ones described in periodic cases. Rabinowitz and Tanaka [11] introduced a type of coercive condition on the 
matrix $L(t)$,

$$
l(t):=\inf _{|u|=1}(L(t) u, u) \rightarrow+\infty \quad \text { as }|t| \rightarrow \infty
$$

and obtained the existence of a homoclinic orbit for non-periodic (1.2) under the Ambrosetti-Rabinowitz $(A R)$ superquadratic condition:

$$
0<\mu H(t, u) \leq\left(H_{u}(t, u), u\right), \quad \forall t \in \mathbb{R}, \forall u \in \mathbb{R}^{N} \backslash\{0\},
$$

where $\mu>2$ is a constant, $(\cdot, \cdot)$ denotes the standard inner product in $\mathbb{R}^{N}$ and the associated norm is denoted by $|\cdot|$.

We should mention that in the case where $M \neq 0$, i.e., the damped vibration system (1.1), only a few authors have studied homoclinic orbits of (1.1); see [19-23]. Zhu [23] considered the periodic case of (1.1) (i.e., $L(t)$ and $H(t, u)$ are $T$-periodic in $t$ with $T>0$ ) and obtained the existence of nontrivial homoclinic solutions of (1.1). The authors [1922] considered the non-periodic case of (1.1): Zhang and Yuan [22] obtained the existence of at least one homoclinic orbit for (1.1) when $H$ satisfies the subquadratic condition at infinity by using a standard minimizing argument; by a symmetric mountain pass theorem and a generalized mountain pass theorem, Wu and Zhang [21] obtained the existence and multiplicity of homoclinic orbits for (1.1) when $H$ satisfies the local (AR) superquadratic growth condition:

$$
0<\mu H(t, u) \leq\left(H_{u}(t, u), u\right), \quad \forall t \in \mathbb{R}, \forall|u| \geq r,
$$

where $\mu>2$ and $r>0$ are two constants. Notice that the authors [21,22] all used condition (1.3). Recently, the author in $[19,20]$ obtained infinitely many homoclinic orbits for (1.1) when $H$ satisfies the subquadratic [19] and asymptotically quadratic [20] condition at infinity by the following weaker conditions than (1.3):

$\left(\mathrm{L}_{1}\right)$ There is a constant $\beta>1$ such that

$$
\operatorname{meas}\left\{t \in \mathbb{R}:|t|^{-\beta} L(t)<b I_{N}\right\}<+\infty, \quad \forall b>0
$$

$\left(\mathrm{L}_{2}\right)$ There is a constant $\gamma_{0} \geq 0$ such that

$$
l(t):=\inf _{|u|=1}(L(t) u, u) \geq-\gamma_{0}, \quad \forall t \in \mathbb{R},
$$

which were firstly used in [15]. It is not hard to check that the matrix-valued function $L(t):=\left(t^{4} \sin ^{2} t+1\right) I_{N}$ satisfies $\left(\mathrm{L}_{1}\right)$ and $\left(\mathrm{L}_{2}\right)$, but does not satisfy (1.3).

We define an operator $\Gamma: H^{1}\left(\mathbb{R}, \mathbb{R}^{N}\right) \rightarrow H^{1}\left(\mathbb{R}, \mathbb{R}^{N}\right)$ by

$$
(\Gamma u, v):=\int_{\mathbb{R}}(M u(t), \dot{v}(t)) d t, \quad \forall u, v \in H^{1}\left(\mathbb{R}, \mathbb{R}^{N}\right) .
$$

Since $M$ is an antisymmetric $N \times N$ constant matrix, $\Gamma$ is self-adjoint on $H^{1}\left(\mathbb{R}, \mathbb{R}^{N}\right)$. Let $\chi$ denote the self-adjoint extension of the operator $-\frac{d^{2}}{d t^{2}}+L(t)+\Gamma$. We are interested in the indefinite case: 
$\left(\mathrm{J}_{1}\right) \quad a:=\sup (\sigma(\chi) \cap(-\infty, 0))<0<b:=\inf (\sigma(\chi) \cap(0, \infty))$.

To state our main result, we still need the following assumptions:

$\left(\mathrm{H}_{1}\right) \quad|\nabla H(t, u)| \leq c\left(1+|u|^{p-1}\right)$ for some $c>0$ and $p>2, \forall t \in \mathbb{R}$ and $u \in \mathbb{R}^{N}$.

$\left(\mathrm{H}_{2}\right) H(t, u) \geq \frac{1}{2} a|u|^{2}, \forall t \in \mathbb{R}$ and $u \in \mathbb{R}^{N}$.

$\left(\mathrm{H}_{3}\right)$ For some $\delta>0$ and $\gamma \in(0, b)$,

$$
|\nabla H(t, u)| \leq \gamma|u|, \quad|H(t, u)| \leq \frac{1}{2}|\nabla H(t, u)| \cdot|u|, \quad \forall|u|<\delta, \forall t \in \mathbb{R} .
$$

$\left(\mathrm{H}_{4}\right) \frac{H(t, u)}{|u|^{2}} \rightarrow+\infty$ as $|u| \rightarrow+\infty$ and there exists $W_{1}(t) \in L^{1}\left(\mathbb{R}, \mathbb{R}^{+}\right)$such that

$$
H(t, u) \geq-W_{1}(t), \quad \forall t \in \mathbb{R} \text { and } u \in \mathbb{R}^{N}
$$

$\left(\mathrm{H}_{5}\right)$ For all $t \in \mathbb{R}$ and $u, z \in \mathbb{R}^{N}$, there holds

$$
\begin{aligned}
& H(t, u+z)-H(t, u)-r(\nabla H(t, u), z)+\frac{(r-1)^{2}}{2}(\nabla H(t, u), u) \\
& \geq-W_{1}(t), \quad \forall r \in[0,1] .
\end{aligned}
$$

Our main results read as follows.

Theorem 1.1 If $\left(\mathrm{L}_{1}\right)-\left(\mathrm{L}_{2}\right),\left(\mathrm{J}_{1}\right)$ and $\left(\mathrm{H}_{1}\right)-\left(\mathrm{H}_{5}\right)$ hold, then (1.1) has at least one nontrivial homoclinic orbit.

Theorem 1.2 Let $\mathcal{M}$ be the collection of solutions of (1.1), then there is a solution that minimizes the energy functional

$$
I(u)=\frac{1}{2} \int_{\mathbb{R}}\left[|\dot{u}(t)|^{2}+(M u(t), \dot{u}(t))+(L(t) u(t), u(t))\right] d t-\int_{\mathbb{R}} H(t, u) d t, \quad u \in E
$$

over $\mathcal{M}$, where the space $E$ is defined in Section 2. In addition, if

$$
|\nabla H(t, u)|=o(|u|) \quad \text { as }|u| \rightarrow 0
$$

uniformly in $t$, then there is a nontrivial homoclinic orbit that minimizes the energy functional over $\mathcal{M} \backslash\{0\}$, i.e., a ground state homoclinic orbit.

Remark 1.1 Although the authors [21] have studied (1.1) with superquadratic nonlinearities, our superquadratic condition $\left(\mathrm{H}_{4}\right)$ is weaker than (1.4) in [21]. Moreover, we study the ground state homoclinic orbit of (1.1). To the best of our knowledge, there has been no result published concerning the ground state homoclinic orbit of (1.1).

\section{Example 1.1}

(1) $H(t, u)=|u|^{p}$,

(2) $H(t, u)=g(t)\left(|u|^{p}+(p-2)|u|^{p-\varepsilon} \sin ^{2}\left(\frac{|u|^{\varepsilon}}{\varepsilon}\right)\right)$,

where $p>2, g(t)>0$ is continuous and $0<\varepsilon<p-2$. It is easy to check that the above two functions satisfy assumptions $\left(\mathrm{H}_{1}\right)-\left(\mathrm{H}_{5}\right)$ if we take $0 \leq W_{1}(t) \in L^{1}\left(\mathbb{R}, \mathbb{R}^{+}\right)$, where $W_{1}(t)$ is the function in $\left(\mathrm{H}_{4}\right)-\left(\mathrm{H}_{5}\right)$. 
The rest of the present paper is organized as follows. In Section 2, we establish the variational framework associated with (1.1), and we also give some preliminary lemmas, which are useful in the proofs of our main results. In Section 3, we give the detailed proofs of our main results.

\section{Preliminary lemmas}

In the following, we use $\|\cdot\|_{L^{p}}$ to denote the norm of $L^{p}\left(\mathbb{R}, \mathbb{R}^{N}\right)$ for any $p \in[1, \infty]$. Let $W:=H^{1}\left(\mathbb{R}, \mathbb{R}^{N}\right)$ be a Hilbert space with the inner product and norm given respectively by

$$
\langle u, v\rangle_{W}=\int_{\mathbb{R}}[(\dot{u}(t), \dot{v}(t))+(u(t), v(t))] d t, \quad\|u\|_{W}=\langle u, u\rangle_{E}^{1 / 2}, \quad \forall u, v \in W .
$$

It is well known that $W$ is continuously embedded in $L^{p}\left(\mathbb{R}, \mathbb{R}^{N}\right)$ for $p \in[2, \infty)$. We define an operator $\Gamma: W \rightarrow W$ by

$$
(\Gamma u, v):=\int_{\mathbb{R}}(M u(t), \dot{v}(t)) d t, \quad \forall u, v \in W .
$$

Since $M$ is an antisymmetric $N \times N$ constant matrix, $\Gamma$ is self-adjoint on $W$. Moreover, we denote by $\chi$ the self-adjoint extension of the operator $-\frac{d^{2}}{d t^{2}}+L(t)+\Gamma$ with the domain $\mathcal{D}(\chi) \subset L^{2}\left(\mathbb{R}, \mathbb{R}^{N}\right)$.

Let $E:=\mathcal{D}\left(|\chi|^{1 / 2}\right)$, the domain of $|\chi|^{1 / 2}$. We define respectively on $E$ the inner product and the norm

$$
\langle u, v\rangle_{E}:=\left(|\chi|^{1 / 2} u,|\chi|^{1 / 2} v\right)_{2}+(u, v)_{2} \quad \text { and } \quad\|u\|_{E}=\langle u, u\rangle_{E}^{1 / 2}
$$

where $(\cdot, \cdot)_{2}$ denotes the inner product in $L^{2}\left(\mathbb{R}, \mathbb{R}^{N}\right)$.

By a similar proof of Lemma 3.1 in [15], we can prove that if conditions $\left(\mathrm{L}_{1}\right)$ and $\left(\mathrm{L}_{2}\right)$ hold, then

$$
E \text { is compactly embedded into } L^{p}\left(\mathbb{R}, \mathbb{R}^{N}\right), \quad \forall p \in[1,+\infty] .
$$

Therefore, it is easy to prove that the spectrum $\sigma(\chi)$ has a sequence of eigenvalues (counted with their multiplicities)

$$
\lambda_{1} \leq \lambda_{2} \leq \cdots \leq \lambda_{k} \leq \cdots \rightarrow \infty
$$

and the corresponding system of eigenfunctions $\left\{e_{k}: k \in \mathbb{N}\right\}\left(\chi e_{k}=\lambda_{k} e_{k}\right)$ forms an orthogonal basis in $L^{2}\left(\mathbb{R}, \mathbb{R}^{N}\right)$.

By $\left(\mathrm{J}_{1}\right)$, we may let

$$
k_{1}:=\sharp\left\{j: \lambda_{j}<0\right\}, \quad E^{-}:=\operatorname{span}\left\{e_{1}, \ldots, e_{k_{1}}\right\}, \quad E^{+}:=\operatorname{cl}_{E}\left(\operatorname{span}\left\{e_{k_{1}+1}, \ldots\right\}\right) .
$$

Then one has the orthogonal decomposition

$$
E=E^{-} \oplus E^{+}
$$

with respect to the inner product $\langle\cdot, \cdot\rangle_{E}$. Now, we introduce respectively on $E$ the following new inner product and norm:

$$
\langle u, v\rangle:=\left(|\chi|^{1 / 2} u,|\chi|^{1 / 2} v\right)_{2}, \quad\|u\|=\langle u, u\rangle^{1 / 2},
$$


where $u, v \in E=E^{-} \oplus E^{+}$with $u=u^{-}+u^{+}$and $v=v^{-}+v^{+}$. Clearly, the norms $\|\cdot\|$ and $\|\cdot\|_{E}$ are equivalent (see [4]), and the decomposition $E=E^{-} \oplus E^{+}$is also orthogonal with respect to both inner products $\langle\cdot, \cdot\rangle$ and $(\cdot, \cdot)_{2}$. Hence, by $\left(J_{1}\right), E$ with equivalent norms, besides, we have

$$
-\left\|u^{-}\right\|^{2}=\left(\chi u^{-}, u^{-}\right)_{2} \leq a\left\|u^{-}\right\|_{L^{2}}^{2}, \quad \forall u^{-} \in E^{-}
$$

and

$$
\left\|u^{+}\right\|^{2}=\left(\chi u^{+}, u^{+}\right)_{2} \geq b\left\|u^{+}\right\|_{L^{2}}^{2}, \quad \forall u^{+} \in E^{+},
$$

where $a$ and $b$ are defined in $\left(\mathrm{J}_{1}\right)$.

For problem (1.1), we consider the following functional:

$$
I(u)=\frac{1}{2} \int_{\mathbb{R}}\left[|\dot{u}(t)|^{2}+(M u(t), \dot{u}(t))+(L(t) u(t), u(t))\right] d t-\int_{\mathbb{R}} H(t, u) d t, \quad u \in E .
$$

Then $I$ can be rewritten as

$$
I(u)=\frac{1}{2}\left\|u^{+}\right\|^{2}-\frac{1}{2}\left\|u^{-}\right\|^{2}-\int_{\mathbb{R}} H(t, u) d t, \quad u=u^{-}+u^{+} \in E .
$$

Let $\Psi(u):=\int_{\mathbb{R}} H(t, u) d t$. In view of the assumptions of $H$, we know $I, \Psi \in C^{1}(E, \mathbb{R})$ and the derivatives are given by

$$
\Psi^{\prime}(u) v=\int_{\mathbb{R}}\left(H_{u}(t, u), v\right) d t, \quad I^{\prime}(u) v=\left\langle u^{+}, v^{+}\right\rangle-\left\langle u^{-}, v^{-}\right\rangle-I^{\prime}(u) v
$$

for any $u, v \in E=E^{-} \oplus E^{+}$with $u=u^{-}+u^{+}$and $v=v^{-}+v^{+}$. By the discussion of [24], the (weak) solutions of system (1.1) are the critical points of the $C^{1}$ functional $I: E \rightarrow \mathbb{R}$. Moreover, it is easy to verify that if $u \neq \equiv$ is a solution of (1.1), then $u(t) \rightarrow 0$ and $\dot{u}(t) \rightarrow 0$ as $|t| \rightarrow \infty$ (see Lemma 3.1 in [25]).

The following abstract critical point theorem plays an important role in proving our main result. Let $E$ be a Hilbert space with the norm $\|\cdot\|$ and have an orthogonal decomposition $E=N \oplus N^{\perp}, N \subset E$ is a closed and separable subspace. There exists a norm $|v|_{\omega}$ satisfying $|v|_{\omega} \leq\|v\|$ for all $v \in N$ and inducing a topology equivalent to the weak topology of $N$ on a bounded subset of $N$. For $u=v+w \in E=N \oplus N^{\perp}$ with $v \in N, w \in N^{\perp}$, we define $|u|_{\omega}^{2}=|v|_{\omega}^{2}+\|w\|^{2}$. Particularly, if $u_{n}=v_{n}+w_{n}$ is $\|\cdot\|$-bounded and $u_{n} \stackrel{|\cdot| \omega}{\rightarrow} u$, then $v_{n} \rightarrow v$ weakly in $N, w_{n} \rightarrow w$ strongly in $N^{\perp}, u_{n} \rightarrow v+w$ weakly in $E$ (cf. [26]).

Let $E:=E^{-} \oplus E^{+}, z_{0} \in E^{+}$with $\left\|z_{0}\right\|=1$. Let $N:=E^{-} \oplus \mathbb{R} z_{0}$ and $E_{1}^{+}:=N^{\perp}=\left(E^{-} \oplus \mathbb{R} z_{0}\right)^{\perp}$. For $R>0$, let

$$
Q:=\left\{u:=u^{-}+s z_{0}: s \in \mathbb{R}^{+}, u^{-} \in E^{-},\|u\|<R\right\}
$$

with $p_{0}=s_{0} z_{0} \in Q, s_{0}>0$. We define

$$
D:=\left\{u:=s z_{0}+w^{+}: s \in \mathbb{R}, w^{+} \in E_{1}^{+},\left\|s z_{0}+w^{+}\right\|=s_{0}\right\} .
$$


For $I \in C^{1}(E, \mathbb{R})$, define

$$
\Gamma:=\left\{\begin{array}{l}
h:[0,1] \times \bar{Q} \mapsto E \text { is }|\cdot|_{\omega} \text {-continuous; } \\
h(0, u)=u \text { and } I(h(s, u)) \leq I(u) \text { for all } u \in \bar{Q} ; \\
\text { For any }\left(s_{0}, u_{0}\right) \in[0,1] \times \bar{Q} \text {,there is a }|\cdot|_{\omega} \text {-neighborhood } \\
\\
U_{\left(s_{0}, u_{0}\right)} \text { s.t. }\left\{u-h(t, u):(t, u) \in U_{\left(s_{0}, u_{0}\right)} \cap([0,1] \times \bar{Q})\right\} \subset E_{\text {fin }}
\end{array}\right\},
$$

where $E_{\text {fin }}$ denotes various finite-dimensional subspaces of $E, \Gamma \neq 0$ since $i d \in \Gamma$.

The variant weak linking theorem is as follows.

Lemma 2.1 ([26]) The family of $C^{1}$-functionals $\left\{I_{\lambda}\right\}$ has the form

$$
I_{\lambda}(u):=\lambda K(u)-J(u), \quad \forall \lambda \in\left[1, \lambda_{0}\right]
$$

where $\lambda_{0}>1$. Assume that

(a) $K(u) \geq 0, \forall u \in E, I_{1}=I$;

(b) $|J(u)|+K(u) \rightarrow \infty$ as $\|u\| \rightarrow \infty$;

(c) $I_{\lambda}$ is $|\cdot|_{\omega}$-upper semicontinuous, $I_{\lambda}^{\prime}$ is weakly sequentially continuous on $E$. Moreover, $I_{\lambda}$ maps bounded sets to bounded sets;

(d) $\sup _{\partial Q} I_{\lambda}<\inf _{D} I_{\lambda}, \forall \lambda \in\left[1, \lambda_{0}\right]$.

Then, for almost all $\lambda \in\left[1, \lambda_{0}\right]$, there exists a sequence $\left\{u_{n}\right\}$ such that

$$
\sup _{n}\left\|u_{n}\right\|<\infty, \quad I_{\lambda}^{\prime}\left(u_{n}\right) \rightarrow 0, \quad I_{\lambda}\left(u_{n}\right) \rightarrow c_{\lambda}
$$

where $c_{\lambda}:=\inf _{h \in \Gamma} \sup _{u \in \bar{Q}} I_{\lambda}(h(t, u)) \in\left[\inf _{D} I_{\lambda}, \sup _{\bar{Q}} I_{\lambda}\right]$

In order to apply Lemma 2.1, we shall prove a few lemmas. We pick $\lambda_{0}$ such that $1<\lambda_{0}<$ $\min [2, b / \gamma]$. For $1 \leq \lambda \leq \lambda_{0}$, we consider

$$
I_{\lambda}(u):=\frac{\lambda}{2}\left\|u^{+}\right\|^{2}-\left(\frac{1}{2}\left\|u^{-}\right\|^{2}+\int_{\mathbb{R}} H(t, u(t)) d t\right):=\lambda K(u)-J(u) .
$$

It is easy to see that $I_{\lambda}$ satisfies condition (a) in Lemma 2.1. To see (c), if $u_{n} \stackrel{|\cdot| \omega_{\omega}}{\rightarrow} u$ and $I_{\lambda}\left(u_{n}\right) \geq c$, then $u_{n}^{+} \rightarrow u^{+}$and $u_{n}^{-} \rightarrow u^{-}$in $E, u_{n} \rightarrow u$ a.e. on $\mathbb{R}$, going to a subsequence if necessary. It follows from the weak lower semicontinuity of the norm, Fatou's lemma and the fact $H(t, u)+W_{1}(t) \geq 0$ for all $t \in \mathbb{R}$ and $u \in \mathbb{R}^{N}$ by $(1.5)$ in $\left(\mathrm{H}_{4}\right)$ that

$$
\begin{aligned}
c & \leq \limsup _{n \rightarrow \infty} I_{\lambda}\left(u_{n}\right) \\
& =\limsup _{n \rightarrow \infty}\left[\frac{\lambda}{2}\left\|u_{n}^{+}\right\|^{2}-\left(\frac{1}{2}\left\|u_{n}^{-}\right\|^{2}+\int_{\mathbb{R}}\left(H\left(t, u_{n}\right)+W_{1}(t)\right) d t\right)+\int_{\mathbb{R}} W_{1}(t) d t\right] \\
& \leq \frac{\lambda}{2}\left\|u^{+}\right\|^{2}-\liminf _{n \rightarrow \infty}\left[\frac{1}{2}\left\|u_{n}^{-}\right\|^{2}+\int_{\mathbb{R}}\left(H\left(t, u_{n}\right)+W_{1}(t)\right) d t\right]+\int_{\mathbb{R}} W_{1}(t) d t \\
& \leq \frac{\lambda}{2}\left\|u^{+}\right\|^{2}-\left(\frac{1}{2}\left\|u^{-}\right\|^{2}+\int_{\mathbb{R}} H(t, u) d t\right)=I_{\lambda}(u) .
\end{aligned}
$$

Thus we get $I_{\lambda}(u) \geq c$. It implies that $I_{\lambda}$ is $|\cdot|_{\omega}$-upper semicontinuous. $I_{\lambda}^{\prime}$ is weakly sequentially continuous on $E$ due to [27]. 
Lemma 2.2 Under assumptions of Theorem 1.1, then

$$
J(u)+K(u) \rightarrow \infty \quad \text { as }\|u\| \rightarrow \infty .
$$

Proof By the definition of $I(u)$ and $\left(\mathrm{H}_{4}\right)$, we have

$$
\begin{aligned}
J(u)+K(u) & =\frac{1}{2}\left\|u^{+}\right\|^{2}+\frac{1}{2}\left\|u^{-}\right\|^{2}+\int_{\mathbb{R}} H(t, u(t)) d t \\
& \geq \frac{1}{2}\|u\|^{2}-\int_{\mathbb{R}} W_{1}(t) d t \rightarrow+\infty \quad \text { as }\|u\| \rightarrow \infty,
\end{aligned}
$$

which is due to $W_{1}(t) \in L^{1}\left(\mathbb{R}, \mathbb{R}^{+}\right)$.

Therefore, Lemma 2.2 implies that condition (b) holds. To continue the discussion, we still need to verify condition (d), that is, the following two lemmas.

Lemma 2.3 Under assumptions of Theorem 1.1, there are two positive constants $\epsilon, \rho>0$ such that

$$
I_{\lambda}(u) \geq \epsilon, \quad u \in E^{+},\|u\|=\rho, \lambda \in\left[1, \lambda_{0}\right] .
$$

Proof By $\left(\mathrm{H}_{1}\right),\left(\mathrm{H}_{3}\right),(2.4)$ and the Sobolev embedding theorem, for all $u \in E^{+}$,

$$
\begin{aligned}
I_{\lambda}(u) & \geq \frac{1}{2}\|u\|^{2}-\int_{\mathbb{R}} H(t, u(t)) d t \\
& =\frac{1}{2}\|u\|^{2}-\int_{\{t \in \mathbb{R}:|u|<\delta\}} H(t, u(t)) d t-\int_{\{t \in \mathbb{R}:|u| \geq \delta\}} H(t, u(t)) d t \\
& \geq \frac{1}{2}\|u\|^{2}-\frac{1}{2} \gamma \int_{\{t \in \mathbb{R}:|u|<\delta\}}|u|^{2} d t-c \int_{\{t \in \mathbb{R}:|u| \geq \delta\}}\left(|u|^{p}+|u|\right) d t \\
& \geq \frac{1}{2}\|u\|^{2}-\frac{\gamma}{b} \frac{1}{2}\|u\|^{2}-C\|u\|^{p} \\
& =\frac{1}{2}\|u\|^{2}\left(1-\frac{\gamma}{b}-2 C\|u\|^{p-2}\right), \quad 0 \leq \gamma<b,
\end{aligned}
$$

where $C$ is a positive constant. It implies the conclusion if we take $\|u\|$ sufficiently small.

Lemma 2.4 Under assumptions of Theorem 1.1, then there is an $R>0$ such that

$$
I_{\lambda}(u) \leq 0, \quad u \in \partial Q_{R}, \lambda \in\left[1, \lambda_{0}\right]
$$

where $Q_{R}:=\left\{u:=v+s z_{0}: s \geq 0, v \in E^{-}, z_{0} \in E^{+}\right.$with $\left.\left\|z_{0}\right\|=1,\|u\| \leq R\right\}$.

Proof Suppose by contradiction that there exist $R_{n} \rightarrow \infty, \lambda_{n} \in\left[1, \lambda_{0}\right]$ and $u_{n}=v_{n}+s_{n} z_{0} \in$ $\partial Q_{R}$ such that $I_{\lambda_{n}}\left(u_{n}\right)>0$. If $s_{n}=0$, then by $\left(\mathrm{H}_{2}\right)$ and (2.3), we have

$$
I_{\lambda_{n}}\left(v_{n}\right)=-\frac{1}{2}\left\|v_{n}\right\|^{2}-\int_{\mathbb{R}} H\left(t, v_{n}\right) d t \leq-\frac{1}{2}\left\|v_{n}\right\|^{2}-\frac{1}{2} a\left\|v_{n}\right\|_{L^{2}}^{2} \leq 0 .
$$


Therefore, $s_{n} \neq 0$ and $\left\|u_{n}\right\|^{2}=\left\|v_{n}\right\|^{2}+s_{n}^{2}=R_{n}^{2}$. Let $\tilde{u}_{n}=\frac{u_{n}}{\left\|u_{n}\right\|}=\tilde{s}_{n} z_{0}+\tilde{v}_{n}$, then

$$
\left\|\tilde{u}_{n}\right\|^{2}=\left\|\tilde{v}_{n}\right\|^{2}+\tilde{s}_{n}^{2}=1
$$

It follows from $I_{\lambda_{n}}\left(u_{n}\right)>0$ and the definition of $I$ that

$$
\begin{aligned}
0 & <\frac{I_{\lambda_{n}}\left(u_{n}\right)}{\left\|u_{n}\right\|^{2}}=\frac{1}{2}\left(\lambda_{n} \tilde{s}_{n}^{2}-\left\|\tilde{v}_{n}\right\|^{2}\right)-\int_{\mathbb{R}} \frac{H\left(t, u_{n}\right)}{\left|u_{n}\right|^{2}}\left|\tilde{u}_{n}\right|^{2} d t \\
& =\frac{1}{2}\left[\left(\lambda_{n}+1\right) \tilde{s}_{n}^{2}-1\right]-\int_{\mathbb{R}} \frac{H\left(t, u_{n}\right)}{\left|u_{n}\right|^{2}}\left|\tilde{u}_{n}\right|^{2} d t .
\end{aligned}
$$

There are renamed subsequences such that $\tilde{s}_{n} \rightarrow \tilde{s}, \lambda_{n} \rightarrow \lambda$, and there is a renamed subsequence such that $\tilde{u}_{n}=\frac{u_{n}}{\left\|u_{n}\right\|}=\tilde{s}_{n} z_{0}+\tilde{v}_{n} \rightarrow \tilde{u}$ in $E$ and $\tilde{u}_{n} \rightarrow \tilde{u}$ a.e. on $\mathbb{R}$.

We claim that

$$
\liminf _{n \rightarrow \infty} \int_{\mathbb{R}} \frac{H\left(t, u_{n}\right)}{\left|u_{n}\right|^{2}}\left|\tilde{u}_{n}\right|^{2} d t \geq 0
$$

Case 1. If $\tilde{u} \not \equiv 0$. Let $\Omega_{0}$ be the subset of $\mathbb{R}$ where $\tilde{u} \neq 0$, then for all $t \in \Omega_{0}$ we have $\left|u_{n}\right|=\left|\tilde{u}_{n}\right| \cdot\left\|u_{n}\right\| \rightarrow \infty$. It follows from $\left(\mathrm{H}_{4}\right)$ and $W_{1}(t) \in L^{1}\left(\mathbb{R}, \mathbb{R}^{+}\right)$that

$$
\int_{\mathbb{R}} \frac{H\left(t, u_{n}\right)}{\left|u_{n}\right|^{2}}\left|\tilde{u}_{n}\right|^{2} d t \geq \int_{\Omega_{0}} \frac{H\left(t, u_{n}\right)}{\left|u_{n}\right|^{2}}\left|\tilde{u}_{n}\right|^{2} d t-\int_{\mathbb{R} \backslash \Omega_{0}} \frac{W_{1}(t)}{\left\|u_{n}\right\|^{2}} d t \rightarrow+\infty \quad \text { as } n \rightarrow \infty
$$

Case 2. If $\tilde{u} \equiv 0$, then by $\left(\mathrm{H}_{4}\right)$ and $W_{1}(t) \in L^{1}\left(\mathbb{R}, \mathbb{R}^{+}\right)$, we have

$$
\int_{\mathbb{R}} \frac{H\left(t, u_{n}\right)}{\left|u_{n}\right|^{2}}\left|\tilde{u}_{n}\right|^{2} d t=\int_{\mathbb{R}} \frac{H\left(t, u_{n}\right)}{\left\|u_{n}\right\|^{2}} d t \geq-\int_{\mathbb{R}} \frac{W_{1}(t)}{\left\|u_{n}\right\|^{2}} d t \rightarrow 0 \quad \text { as } n \rightarrow \infty
$$

Therefore, Cases 1 and 2 imply that (2.6) holds. Therefore, by (2.5), (2.6) and the facts $\tilde{s}_{n} \rightarrow \tilde{s}, \lambda_{n} \rightarrow \lambda$, we have

$$
(\lambda+1) \tilde{s}^{2}-1 \geq 0
$$

that is, $\tilde{s}^{2} \geq \frac{1}{1+\lambda} \geq \frac{1}{1+\lambda_{0}}>0$. Thus, $\tilde{u} \neq 0$. It follows from $\left(\mathrm{H}_{4}\right)$ that

$$
\int_{\mathbb{R}} \frac{H\left(t, u_{n}\right)}{\left|u_{n}\right|^{2}}\left|\tilde{u}_{n}\right|^{2} d t \rightarrow+\infty \quad \text { as } n \rightarrow \infty
$$

which contradicts (2.5). The proof is finished.

Therefore, Lemmas 2.3 and 2.4 imply that condition (d) of Lemma 2.1 holds. Applying Lemma 2.1, we soon obtain the following fact.

Lemma 2.5 Under assumptions of Theorem 1.1, for almost all $\lambda \in\left[1, \lambda_{0}\right]$, there exists a sequence $\left\{u_{n}\right\}$ such that

$$
\sup _{n}\left\|u_{n}\right\|<\infty, \quad I_{\lambda}^{\prime}\left(u_{n}\right) \rightarrow 0, \quad I_{\lambda}\left(u_{n}\right) \rightarrow c_{\lambda}
$$

where the definition of $c_{\lambda}$ is given in Lemma 2.1. 
Lemma 2.6 Under assumptions of Theorem 1.1, for almost all $\lambda \in\left[1, \lambda_{0}\right]$, there exists a $u_{\lambda} \in E$ such that

$$
I_{\lambda}^{\prime}\left(u_{\lambda}\right)=0, \quad I_{\lambda}\left(u_{\lambda}\right)=c_{\lambda} .
$$

Proof Let $\left\{u_{n}\right\}$ be the sequence obtained in Lemma 2.5. Since $\left\{u_{n}\right\}$ is bounded, we can assume $u_{n} \rightarrow u_{\lambda}$ in $E$ and $u_{n} \rightarrow u_{\lambda}$ a.e. on $\mathbb{R}$. By $\left(\mathrm{H}_{1}\right),\left(\mathrm{H}_{3}\right),(2.1)$ and Theorem A.4 in [27], we have

$$
\int_{\mathbb{R}} \frac{1}{2}\left(\nabla H\left(t, u_{n}\right), u_{n}\right) d t \rightarrow \int_{\mathbb{R}} \frac{1}{2}\left(\nabla H\left(t, u_{\lambda}\right), u_{\lambda}\right) d t
$$

and

$$
\int_{\mathbb{R}} H\left(t, u_{n}\right) d t \rightarrow \int_{\mathbb{R}} H\left(t, u_{\lambda}\right) d t .
$$

By Lemma 2.5 and the fact that $I_{\lambda}^{\prime}$ is weakly sequentially continuous, we have

$$
I_{\lambda}^{\prime}\left(u_{\lambda}\right) \varphi=\lim _{n \rightarrow \infty} I_{\lambda}^{\prime}\left(u_{n}\right) \varphi=0, \quad \forall \varphi \in E
$$

That is, $I_{\lambda}^{\prime}\left(u_{\lambda}\right)=0$. By Lemma 2.5 , we have

$$
I_{\lambda}\left(u_{n}\right)-\frac{1}{2} I_{\lambda}^{\prime}\left(u_{n}\right) u_{n}=\int_{\mathbb{R}}\left(\frac{1}{2}\left(\nabla H\left(t, u_{n}\right), u_{n}\right)-H\left(t, u_{n}\right)\right) d t \rightarrow c_{\lambda} .
$$

It follows from (2.7), (2.8) and the fact $I_{\lambda}^{\prime}\left(u_{\lambda}\right)=0$ that

$$
I_{\lambda}\left(u_{\lambda}\right)=I_{\lambda}\left(u_{\lambda}\right)-\frac{1}{2} I_{\lambda}^{\prime}\left(u_{\lambda}\right) u_{\lambda}=\int_{\mathbb{R}}\left(\frac{1}{2}\left(\nabla H\left(t, u_{\lambda}\right), u_{\lambda}\right)-H\left(t, u_{\lambda}\right)\right) d t=c_{\lambda} .
$$

The proof is finished.

Applying Lemma 2.6, we soon obtain the following fact.

Lemma 2.7 Under assumptions of Theorem 1.1, for every $\lambda \in\left[1, \lambda_{0}\right]$, there are sequences $\left\{u_{n}\right\} \subset E$ and $\lambda_{n} \in\left[1, \lambda_{0}\right]$ with $\lambda_{n} \rightarrow \lambda$ such that

$$
I_{\lambda_{n}}^{\prime}\left(u_{n}\right)=0, \quad I_{\lambda_{n}}\left(u_{n}\right)=c_{\lambda_{n}} .
$$

Lemma 2.8 Under assumptions of Theorem 1.1, then

$$
\int_{\mathbb{R}}\left[H(t, u)-H(t, r w)+r^{2}(\nabla H(t, u), w)-\frac{1+r^{2}}{2}(\nabla H(t, u), u)\right] d t \leq C,
$$

where $u \in E, w \in E^{+}, 0 \leq r \leq 1$ and the constant $C:=\int_{\mathbb{R}}\left|W_{1}(t)\right| d t$ does not depend on $u$, $w, r$.

Proof This follows from $\left(\mathrm{H}_{5}\right)$ if we take $u=u$ and $z=r w-u$. 
Lemma 2.9 The sequences given in Lemma 2.7 are bounded.

Proof Write $u_{n}=u_{n}^{+}+u_{n}^{-}$, where $u_{n}^{ \pm} \in E^{ \pm}$. Suppose that

$$
\left\|u_{n}\right\| \rightarrow \infty \quad \text { as } n \rightarrow \infty .
$$

Let $v_{n}:=\frac{u_{n}}{\left\|u_{n}\right\|}$, then $v_{n}^{+}=\frac{u_{n}^{+}}{\left\|u_{n}\right\|}, v_{n}^{-}=\frac{u_{n}^{-}}{\left\|u_{n}\right\|},\left\|v_{n}\right\|^{2}=\left\|v_{n}^{+}\right\|^{2}+\left\|v_{n}^{-}\right\|^{2}=1$ and $\left\|v_{n}^{+}\right\| \leq 1$. Thus $v_{n}^{+} \rightarrow v^{+}$in $E$ and $v_{n}^{+} \rightarrow v^{+}$a.e. on $\mathbb{R}$, after passing to a subsequence.

Case 1 . If $v^{+} \not \equiv 0$. Let $\Omega_{1}$ be the subset of $\mathbb{R}$ where $v^{+} \neq 0$. Then $v \neq 0$ and $\left|u_{n}\right|=\left|v_{n}\right|$. $\left\|u_{n}\right\| \rightarrow \infty$ on $\Omega_{1}$. It follows from $\left(\mathrm{H}_{4}\right)$ and $W_{1}(t) \in L^{1}\left(\mathbb{R}, \mathbb{R}^{+}\right)$that

$$
\int_{\mathbb{R}} \frac{H\left(t, u_{n}\right)}{\left|u_{n}\right|^{2}}\left|v_{n}\right|^{2} d t \geq \int_{\Omega_{1}} \frac{H\left(t, u_{n}\right)}{\left|u_{n}\right|^{2}}\left|v_{n}\right|^{2} d t-\int_{\mathbb{R} \backslash \Omega_{1}} \frac{W_{1}(t)}{\left\|u_{n}\right\|^{2}} d t \rightarrow+\infty \quad \text { as } n \rightarrow \infty,
$$

which together with Lemmas 2.3 and 2.7 and $v_{n}^{ \pm} \rightarrow v^{ \pm}$in $L^{q}\left(\mathbb{R}, \mathbb{R}^{N}\right)$ for all $1 \leq q \leq \infty$ (by (2.1)) implies that

$$
0 \leq \frac{c_{\lambda_{n}}}{\left\|u_{n}\right\|^{2}}=\frac{I_{\lambda_{n}}\left(u_{n}\right)}{\left\|u_{n}\right\|^{2}}=\frac{\lambda_{n}}{2}\left\|v_{n}^{+}\right\|^{2}-\frac{1}{2}\left\|v_{n}^{-}\right\|^{2}-\int_{\mathbb{R}} \frac{H\left(t, u_{n}\right)}{\left|u_{n}\right|^{2}}\left|v_{n}\right|^{2} d t \rightarrow-\infty \quad \text { as } n \rightarrow \infty .
$$

It is a contradiction.

Case 2. If $v^{+} \equiv 0$. We claim that there is a constant $C$ independent of $u_{n}$ and $\lambda_{n}$ such that

$$
I_{\lambda_{n}}\left(r u_{n}^{+}\right)-I_{\lambda_{n}}\left(u_{n}\right) \leq C, \quad \forall r \in[0,1] .
$$

Since

$$
\frac{1}{2} I_{\lambda_{n}}^{\prime}\left(u_{n}\right) \varphi=\frac{1}{2} \lambda_{n}\left\langle u_{n}^{+}, \varphi^{+}\right\rangle-\frac{1}{2}\left\langle u_{n}^{-}, \varphi^{-}\right\rangle-\frac{1}{2} \int_{\mathbb{R}}\left(\nabla H\left(t, u_{n}\right), \varphi\right) d t=0, \quad \forall \varphi \in E,
$$

it follows from the definition of $I$ that

$$
\begin{aligned}
I_{\lambda_{n}}\left(r u_{n}^{+}\right)-I_{\lambda_{n}}\left(u_{n}\right)= & \frac{1}{2} \lambda_{n}\left(r^{2}-1\right)\left\|u_{n}^{+}\right\|^{2}+\frac{1}{2}\left\|u_{n}^{-}\right\|^{2}+\int_{\mathbb{R}}\left(H\left(t, u_{n}\right)-H\left(t, r u_{n}^{+}\right)\right) d t \\
& +\frac{1}{2} \lambda_{n}\left\langle u_{n}^{+}, \varphi^{+}\right\rangle-\frac{1}{2}\left\langle u_{n}^{-}, \varphi^{-}\right\rangle-\frac{1}{2} \int_{\mathbb{R}}\left(\nabla H\left(t, u_{n}\right), \varphi\right) d t .
\end{aligned}
$$

Take $\varphi:=\left(r^{2}+1\right) u_{n}^{-}-\left(r^{2}-1\right) u_{n}^{+}=\left(r^{2}+1\right) u_{n}-2 r^{2} u_{n}^{+}$in $(2.10)$, then it follows from Lemma 2.8 that

$$
\begin{aligned}
I_{\lambda_{n}}\left(r u_{n}^{+}\right)-I_{\lambda_{n}}\left(u_{n}\right)= & -\frac{r^{2}}{2}\left\|u_{n}^{-}\right\|^{2}+\int_{\mathbb{R}}\left[H\left(t, u_{n}\right)-H\left(t, r u_{n}^{+}\right)+r^{2}\left(\nabla H\left(t, u_{n}\right), u_{n}^{+}\right)\right. \\
& \left.-\frac{1+r^{2}}{2}\left(\nabla H\left(t, u_{n}\right), u_{n}\right)\right] d t \\
\leq & C .
\end{aligned}
$$

Thus (2.9) holds.

Let $C_{0}>0$ be a fixed constant and take

$$
r_{n}:=\frac{C_{0}}{\left\|u_{n}\right\|} \rightarrow 0 \quad \text { as } n \rightarrow \infty .
$$


Therefore, (2.9) implies that

$$
I_{\lambda_{n}}\left(r_{n} u_{n}^{+}\right)-I_{\lambda_{n}}\left(u_{n}\right) \leq C
$$

It follows from $v_{n}^{+}=\frac{u_{n}^{+}}{\left\|u_{n}\right\|}$ and Lemma 2.7 that

$$
I_{\lambda_{n}}\left(C_{0} v_{n}^{+}\right) \leq C^{\prime}
$$

Note that Lemmas 2.3 and 2.7 and $\left(\mathrm{H}_{4}\right)$ imply that

$$
\begin{aligned}
0 & \leq \frac{c_{\lambda_{n}}}{\left\|u_{n}\right\|^{2}}=\frac{I_{\lambda_{n}}\left(u_{n}\right)}{\left\|u_{n}\right\|^{2}}=\frac{\lambda_{n}}{2}\left\|v_{n}^{+}\right\|^{2}-\frac{1}{2}\left\|v_{n}^{-}\right\|^{2}-\frac{\int_{\mathbb{R}} H\left(t, u_{n}\right)}{\left\|u_{n}\right\|^{2}} d t \\
& \leq \frac{\lambda_{0}}{2}\left\|v_{n}^{+}\right\|^{2}-\frac{1}{2}\left\|v_{n}^{-}\right\|^{2}+\frac{\int_{\mathbb{R}} W_{1}(t) d t}{\left\|u_{n}\right\|^{2}} .
\end{aligned}
$$

It follows from the fact $\frac{\int_{\mathbb{R}} W_{1}(t) d t}{\left\|u_{n}\right\|^{2}} \rightarrow 0$ as $n \rightarrow \infty$ due to $W_{1}(t) \in L^{1}\left(\mathbb{R}, \mathbb{R}^{+}\right)$that

$$
\frac{\lambda_{0}}{2}\left\|v_{n}^{+}\right\|^{2}-\frac{1}{2}\left\|v_{n}^{-}\right\|^{2}+\varepsilon \geq 0, \quad \forall \varepsilon>0
$$

for all sufficiently large $n$. We take $\varepsilon=\frac{1}{4}$, by (2.12) and $\left\|v_{n}\right\|^{2}=\left\|v_{n}^{+}\right\|^{2}+\left\|v_{n}^{-}\right\|^{2}=1$, we have

$$
\left\|v_{n}^{+}\right\|^{2} \geq \frac{1}{2\left(1+\lambda_{0}\right)}
$$

for all sufficiently large $n$. By $\left(\mathrm{H}_{1}\right)$ and $\left(\mathrm{H}_{3}\right)$, we have

$$
\begin{aligned}
& \int_{\mathbb{R}} H\left(t, C_{0} v_{n}^{+}\right) d t \\
& \quad \leq \frac{1}{2} \gamma C_{0}^{2} \int_{\left\{t \in \mathbb{R}:\left|C_{0} v_{n}^{+}\right|<\delta\right\}}\left|v_{n}^{+}\right|^{2} d t+\frac{1}{2} c \int_{\left\{t \in \mathbb{R}:\left|C_{0} v_{n}^{+}\right| \geq \delta\right\}}\left(C_{0}\left|v_{n}^{+}\right|+C_{0}^{p}\left|v_{n}^{+}\right|^{p}\right) d t \\
& \quad \leq \frac{1}{2} \gamma C_{0}^{2} \int_{\left\{t \in \mathbb{R}:\left|C_{0} v_{n}^{+}\right|<\delta\right\}}\left|v_{n}^{+}\right|^{2} d t+C_{1} C_{0}^{p} \int_{\left\{t \in \mathbb{R}:\left|C_{0} v_{n}^{+}\right| \geq \delta\right\}}\left|v_{n}^{+}\right|^{p} d t .
\end{aligned}
$$

For all sufficiently large $n$, by (2.13) and (2.14), it follows from $\lambda_{n} \rightarrow \lambda$ and $v_{n}^{+} \rightarrow v^{+} \equiv 0$ in $L^{q}\left(\mathbb{R}, \mathbb{R}^{N}\right)$ for all $1 \leq q \leq \infty$ (by $\left.(2.1)\right)$ that

$$
\begin{aligned}
I_{\lambda_{n}}\left(C_{0} v_{n}^{+}\right)= & \frac{1}{2} \lambda_{n} C_{0}^{2}\left\|v_{n}^{+}\right\|^{2}-\int_{\mathbb{R}} H\left(t, C_{0} v_{n}^{+}\right) d t \\
\geq & \frac{1}{2} \lambda_{n} C_{0}^{2} \frac{1}{2\left(1+\lambda_{0}\right)}-\frac{1}{2} \gamma C_{0}^{2} \int_{\left\{t \in \mathbb{R}:\left|C_{0} v_{n}^{+}\right|<\delta\right\}}\left|v_{n}^{+}\right|^{2} d t \\
& -C_{1} C_{0}^{p} \int_{\left\{t \in \mathbb{R}:\left|C_{0} v_{n}^{+}\right| \geq \delta\right\}}\left|v_{n}^{+}\right|^{p} d t \\
\rightarrow & \frac{\lambda C_{0}^{2}}{4\left(1+\lambda_{0}\right)} \text { as } n \rightarrow \infty .
\end{aligned}
$$

This implies that $I_{\lambda_{n}}\left(C_{0} v_{n}^{+}\right) \rightarrow \infty$ as $C_{0} \rightarrow \infty$, contrary to (2.11).

Therefore, $\left\{u_{n}\right\}$ are bounded. The proof is finished. 


\section{Proofs of the main results}

Proof of Theorem 1.1 From Lemma 2.7, there are sequences $1<\lambda_{n} \rightarrow 1$ and $\left\{u_{n}\right\} \subset E$ such that $I_{\lambda_{n}}^{\prime}\left(u_{n}\right)=0$ and $I_{\lambda_{n}}\left(u_{n}\right)=c_{\lambda_{n}}$. By Lemma 2.9, we know that $\left\{u_{n}\right\}$ is bounded in $E$. Thus we can assume $u_{n} \rightarrow u$ in $E, u_{n} \rightarrow u$ a.e. on $\mathbb{R}$. Therefore,

$$
I_{\lambda_{n}}^{\prime}\left(u_{n}\right) \varphi=\lambda_{n}\left\langle u_{n}^{+}, \varphi\right\rangle-\left\langle u_{n}^{-}, \varphi\right\rangle-\int_{\mathbb{R}}\left(\nabla H\left(t, u_{n}\right), \varphi\right) d t=0, \quad \forall \varphi \in E .
$$

Hence, in the limit,

$$
I^{\prime}(u) \varphi=\left\langle u^{+}, \varphi\right\rangle-\left\langle u^{-}, \varphi\right\rangle-\int_{\mathbb{R}}(\nabla H(t, u), \varphi) d t=0, \quad \forall \varphi \in E .
$$

Thus $I^{\prime}(u)=0$. Note that

$$
I_{\lambda_{n}}\left(u_{n}\right)-\frac{1}{2} I_{\lambda_{n}}^{\prime}\left(u_{n}\right) u_{n}=\int_{\mathbb{R}}\left(\frac{1}{2}\left(\nabla H\left(t, u_{n}\right), u_{n}\right)-H\left(t, u_{n}\right)\right) d t=c_{\lambda_{n}} \geq c_{1} .
$$

Similar to (2.7) and (2.8), we know

$$
\int_{\mathbb{R}}\left(\frac{1}{2}\left(\nabla H\left(t, u_{n}\right), u_{n}\right)-H\left(t, u_{n}\right)\right) d t \rightarrow \int_{\mathbb{R}}\left(\frac{1}{2}(\nabla H(t, u), u)-H(t, u)\right) d t \quad \text { as } n \rightarrow \infty .
$$

It follows from $I^{\prime}(u)=0,(3.1)$ and Lemma 2.3 that

$$
\begin{aligned}
I(u) & =I(u)-\frac{1}{2} I^{\prime}(u) u=\int_{\mathbb{R}}\left[\frac{1}{2}(\nabla H(t, u), u)-H(t, u)\right] d t \\
& =\lim _{n \rightarrow \infty} \int_{\mathbb{R}}\left(\frac{1}{2}\left(\nabla H\left(t, u_{n}\right), u_{n}\right)-H\left(t, u_{n}\right)\right) d t \geq c_{1} \geq \epsilon>0 .
\end{aligned}
$$

Therefore, $u \neq 0$.

Proof of Theorem 1.2 By Theorem 1.1, $\mathcal{M} \neq \emptyset$, where $\mathcal{M}$ is the collection of solutions of (1.1). Let

$$
\alpha:=\inf _{u \in \mathcal{M}} I(u)
$$

If $u$ is a solution of (1.1), then by Lemma 2.8 (take $r=0$ ),

$$
I(u)=I(u)-\frac{1}{2} I^{\prime}(u) u=\int_{\mathbb{R}}\left[\frac{1}{2}(\nabla H(t, u), u)-H(t, u)\right] d t \geq-C=-\int_{\mathbb{R}}\left|W_{1}(t)\right| d t .
$$

Thus $\alpha>-\infty$. Let $\left\{u_{n}\right\}$ be a sequence in $\mathcal{M}$ such that

$$
I\left(u_{n}\right) \rightarrow \alpha .
$$

By Lemma 2.9, the sequence $\left\{u_{n}\right\}$ is bounded in $E$. Therefore, $u_{n} \rightarrow u$ in $E, u_{n} \rightarrow u$ a.e. on $\mathbb{R}$ and $u_{n} \rightarrow u$ in $L^{p}\left(\mathbb{R}, \mathbb{R}^{N}\right)$ for all $p \in[1,+\infty]$ (by (2.1)), after passing to a subsequence. Therefore,

$$
I^{\prime}\left(u_{n}\right) \varphi=\left\langle u_{n}^{+}, \varphi\right\rangle-\left\langle u_{n}^{-}, \varphi\right\rangle-\int_{\mathbb{R}}\left(\nabla H\left(t, u_{n}\right), \varphi\right) d t=0, \quad \forall \varphi \in E .
$$


Hence, in the limit,

$$
I^{\prime}(u) \varphi=\left\langle u^{+}, \varphi\right\rangle-\left\langle u^{-}, \varphi\right\rangle-\int_{\mathbb{R}}(\nabla H(t, u), \varphi) d t=0, \quad \forall \varphi \in E .
$$

Thus $I^{\prime}(u)=0$. Similar to (2.7) and (2.8), we have

$$
\begin{aligned}
I\left(u_{n}\right)-\frac{1}{2} I^{\prime}\left(u_{n}\right) u_{n} & =\int_{\mathbb{R}}\left(\frac{1}{2}\left(\nabla H\left(t, u_{n}\right), u_{n}\right)-H\left(t, u_{n}\right)\right) d t \\
& \rightarrow \int_{\mathbb{R}}\left(\frac{1}{2}(\nabla H(t, u), u)-H(t, u)\right) d t \quad \text { as } n \rightarrow \infty .
\end{aligned}
$$

It follows from $I^{\prime}(u)=0$ and (3.2) that

$$
\begin{aligned}
I(u) & =I(u)-\frac{1}{2} I^{\prime}(u) u=\int_{\mathbb{R}}\left[\frac{1}{2}(\nabla H(t, u), u)-H(t, u)\right] d t \\
& =\lim _{n \rightarrow \infty} \int_{\mathbb{R}}\left(\frac{1}{2}\left(\nabla H\left(t, u_{n}\right), u_{n}\right)-H\left(t, u_{n}\right)\right) d t \\
& =\lim _{n \rightarrow \infty} I\left(u_{n}\right)=\alpha .
\end{aligned}
$$

Now suppose that

$$
|\nabla H(t, u)|=o(|u|) \quad \text { as }|u| \rightarrow 0
$$

It follows from $\left(\mathrm{H}_{1}\right)$ that for any $\varepsilon>0$, there is a constant $C_{\varepsilon}>0$ such that

$$
|\nabla H(t, u)| \leq \varepsilon|u|+C_{\varepsilon}|u|^{p-1} .
$$

Let

$$
\beta:=\inf _{u \in M^{\prime}} I(u)
$$

where $M^{\prime}:=\mathcal{M} \backslash\{0\}$. Let $\left\{u_{n}\right\}$ be a sequence in $\mathcal{M} \backslash\{0\}$ such that

$$
I\left(u_{n}\right) \rightarrow \beta
$$

Note that

$$
0=I^{\prime}\left(u_{n}\right) u_{n}^{+}=\left\|u_{n}^{+}\right\|^{2}-\int_{\mathbb{R}}\left(\nabla H\left(t, u_{n}\right), u_{n}^{+}\right) d t
$$

which together with (3.3), Hölder's inequality and the Sobolev embedding theorem implies

$$
\begin{aligned}
\left\|u_{n}^{+}\right\|^{2} & =\int_{\mathbb{R}}\left(\nabla H\left(t, u_{n}\right), u_{n}^{+}\right) d t \\
& \leq \varepsilon \int_{0}^{T}\left|u_{n}\right| \cdot\left|u_{n}^{+}\right| d t+C_{\varepsilon} \int_{0}^{T}\left|u_{n}\right|^{p-1}\left|u_{n}^{+}\right| d t \\
& \leq \varepsilon\left\|u_{n}\right\| \cdot\left\|u_{n}^{+}\right\|+C_{\varepsilon}^{\prime}\left\|u_{n}\right\|_{L^{p}}^{p-1}\left\|u_{n}^{+}\right\|
\end{aligned}
$$




$$
\begin{aligned}
& \leq \varepsilon\left\|u_{n}\right\| \cdot\left\|u_{n}^{+}\right\|+C_{\varepsilon}^{\prime \prime}\left\|u_{n}\right\|_{L^{p}}^{p-2}\left\|u_{n}\right\| \cdot\left\|u_{n}^{+}\right\| \\
& \leq \varepsilon\left\|u_{n}\right\|^{2}+C_{\varepsilon}^{\prime \prime}\left\|u_{n}\right\|_{L^{p}}^{p-2}\left\|u_{n}\right\|^{2} .
\end{aligned}
$$

Similarly, we have

$$
\left\|u_{n}^{-}\right\|^{2} \leq \varepsilon\left\|u_{n}\right\|^{2}+C_{\varepsilon}^{\prime \prime}\left\|u_{n}\right\|_{L^{p}}^{p-2}\left\|u_{n}\right\|^{2} .
$$

From (3.5) and (3.6), we get

$$
\left\|u_{n}\right\|^{2} \leq 2 \varepsilon\left\|u_{n}\right\|^{2}+2 C_{\varepsilon}^{\prime \prime}\left\|u_{n}\right\|_{L^{p}}^{p-2}\left\|u_{n}\right\|^{2}
$$

which means $\left\|u_{n}\right\|_{L^{p}} \geq C$ for some constant $C>0$. Since $u_{n} \rightarrow u$ in $L^{p}\left(\mathbb{R}, \mathbb{R}^{N}\right)$, we know $u \neq 0$. As before, $I\left(u_{n}\right) \rightarrow I(u)=\beta$ as $n \rightarrow \infty$.

\section{Competing interests}

The authors declare that they have no competing interests.

\section{Authors' contributions}

The main idea of this paper was proposed by G-WC and G-WC prepared the manuscript initially and JW performed a part of steps of the proofs in this research. All authors read and approved the final manuscript.

\section{Acknowledgements}

The authors thank the referees and the editors for their helpful comments and suggestions. Research was supported by the Tianyuan Fund for Mathematics of NSFC (Grant No. 11326113) and the Key Project of Natural Science Foundation of Educational Committee of Henan Province of China (Grant No. 13A110015).

Received: 6 January 2014 Accepted: 24 April 2014 Published: 09 May 2014

\section{References}

1. Ambrosetti, A, Coti Zelati, V: Multiple homoclinic orbits for a class of conservative systems. Rend. Semin. Mat. Univ. Padova 89, 177-194 (1993)

2. Chen, G, Ma, S: Periodic solutions for Hamiltonian systems without Ambrosetti-Rabinowitz condition and spectrum. J. Math. Anal. Appl. 379, 842-851 (2011)

3. Chen, G, Ma, S: Ground state periodic solutions of second order Hamiltonian systems without spectrum 0. Isr. J. Math 198, 111-127 (2013)

4. Ding, Y: Existence and multiplicity results for homoclinic solutions to a class of Hamiltonian systems. Nonlinear Anal. 25, 1095-1113 (1995)

5. Izydorek, M, Janczewska, J: Homoclinic solutions for a class of second order Hamiltonian systems. J. Differ. Equ. 219 375-389 (2005)

6. Kim, Y: Existence of periodic solutions for planar Hamiltonian systems at resonance. J. Korean Math. Soc. 48, 1143-1152 (2011)

7. Mawhin, J, Willem, M: Critical Point Theory and Hamiltonian Systems. Applied Mathematical Sciences, vol. 74 Springer, New York (1989)

8. Omana, W, Willem, M: Homoclinic orbits for a class of Hamiltonian systems. Differ. Integral Equ. 5, 1115-1120 (1992)

9. Paturel, E: Multiple homoclinic orbits for a class of Hamiltonian systems. Calc. Var. Partial Differ. Equ. 12, 117-143 (2001)

10. Rabinowitz, PH: Homoclinic orbits for a class of Hamiltonian systems. Proc. R. Soc. Edinb., Sect. A 114, 33-38 (1990)

11. Rabinowitz, PH, Tanaka, K: Some results on connecting orbits for a class of Hamiltonian systems. Math. Z. 206, 473-499 (1991)

12. Séré, E: Existence of infinitely many homoclinic orbits in Hamiltonian systems. Math. Z. 209, 133-160 (1992)

13. Sun, J, Chen, H, Nieto, JJ: Homoclinic solutions for a class of subquadratic second-order Hamiltonian systems. J. Math. Anal. Appl. 373, 20-29 (2011)

14. Tang, X, Xiao, L: Homoclinic solutions for non-autonomous second-order Hamiltonian systems with a coercive potential. J. Math. Anal. Appl. 351, 586-594 (2009)

15. Wan, $L$, Tang, C: Existence and multiplicity of homoclinic orbits for second order Hamiltonian systems without (AR) condition. Discrete Contin. Dyn. Syst., Ser. B 15, 255-271 (2011)

16. Xiao, J, Nieto, JJ: Variational approach to some damped Dirichlet nonlinear impulsive differential equations. J. Franklin Inst. 348, 369-377 (2011)

17. Zhang, P, Tang, C: Infinitely many periodic solutions for nonautonomous sublinear second-order Hamiltonian systems. Abstr. Appl. Anal. 2010, 620438 (2010). doi:10.1155/2010/620438

18. Zhang, Q, Liu, C: Infinitely many homoclinic solutions for second order Hamiltonian systems. Nonlinear Anal. 72 894-903 (2010) 
19. Chen, G: Non-periodic damped vibration systems with sublinear terms at infinity: infinitely many homoclinic orbits. Nonlinear Anal. 92, 168-176 (2013)

20. Chen, G: Non-periodic damped vibration systems with asymptotically quadratic terms at infinity: infinitely many homoclinic orbits. Abstr. Appl. Anal. 2013, 937128 (2013)

21. Wu, X, Zhang, W: Existence and multiplicity of homoclinic solutions for a class of damped vibration problems. Nonlinear Anal. 74, 4392-4398 (2011)

22. Zhang, Z, Yuan, R: Homoclinic solutions for some second-order nonautonomous systems. Nonlinear Anal. 71 , 5790-5798 (2009)

23. Zhu, W: Existence of homoclinic solutions for a class of second order systems. Nonlinear Anal. 75, 2455-2463 (2012)

24. Costa, DG, Magalhães, CA: A variational approach to subquadratic perturbations of elliptic systems. J. Differ. Equ. 111، 103-122 (1994)

25. Wang, J, Xu, J, Zhang, F: Homoclinic orbits for a class of Hamiltonian systems with superquadratic or asymptotically quadratic potentials. Commun. Pure Appl. Anal. 10, 269-286 (2011)

26. Schechter, M, Zou, W: Weak linking theorems and Schrödinger equations with critical Sobolev exponent. ESAIM Control Optim. Calc. Var. 9, 601-619 (2003)

27. Willem, M: Minimax Theorems. Birkhäuser, Boston (1996)

10.1186/1687-2770-2014-106

Cite this article as: Chen and Wang: Ground state homoclinic orbits of damped vibration problems. Boundary Value Problems 2014, 2014:106

\section{Submit your manuscript to a SpringerOpen ${ }^{\circ}$ journal and benefit from:}

- Convenient online submission

- Rigorous peer review

- Immediate publication on acceptance

- Open access: articles freely available online

- High visibility within the field

- Retaining the copyright to your article 